\title{
On a Fractional Master Equation and a Fractional Diffusion Equation
}

\author{
R. K. Saxena \\ Department of Mathematics and Statistics, Jai Narain, Vyas University, Jodhpur-342004, India \\ *Corresponding Author: ram.saxena@yahoo.com
}

Copyright $@ 2013$ Horizon Research Publishing All rights reserved.

\begin{abstract}
In this paper, we derive the solutions of fractional master equation defined by (2.1) and fractional diffusion equation defined by (3.3). The method followed in deriving the solution is that of Laplace and Fourier transforms. The solutions are obtained in a neat and compact forms in terms of the generalized Mittag -Leffler function and Fox' H-function. The results established are of general character and include some known results, as special cases.
\end{abstract}

Keywords Fractional Master Equation, Laplace Transform, Fourier Transform, Generalized Mittag -Leffler Function, Fox's H-Function And Fourier Space

\section{Introduction}

Fractional Master equations are studied in order to explain certain physical phenomena arising in science and engineering. Fractional Master equation and fractional random walks are discussed by Hilfer and Anton [1]. Fractional Master equation for non- standard analysis associated with Riemann-Liouville derivative is discussed by Jumarie [2]. A generalized master equation is constructed from non- homogeneous random walk scheme by Pagnini, Mura and Mainardi [3]. Fractional Fokker-Planck equations are derived from generalized fractional Master equation by Metzler, Barkai and Klafter [4].

In a recent paper El-Wakil and Zahran [5] obtained a relation between CTRW and fractional master equation by obtaining the corresponding waiting time density in terms of generalized Mittag-Leffler function [6]. The solution of the master equation is derived by them in Fourier space. The object of this paper is to obtain the complete solution of the master equation in a closed form in terms of the Mittag-Leffler function and the

H-function [7]. Furthermore, an alternative shorter and simple method based on a result obtained by the Saxena, Mathai and Haubold [8] is given to derive the solution of a generalized fractional diffusion equation.

On the basis of a classification of the time generators in ergodic theory, Hilfer and Anton [1] introduced the fractional master equation involving fractional time derivative of order $\alpha,(0<\alpha \leq 1)$. Fractional master equations occur as special cases of generalized Liouville equations, see Hilfer $[9,10]$ and provide a generalization of the fractional diffusion. It is proved by Hilfer and Anton [1] that there exists a interesting relation between a fractal master equation and the theory of continuous random walks.

Following Hilfer and Anton [1,p.R848)], fractional master equation can be written formally as

$$
{ }_{0} D_{t}^{\alpha} N(r, t)=\sum_{r^{\prime}} W\left(r-r^{\prime}\right) N\left(r^{\prime}, t\right), 0<\alpha<1,(1.1)
$$

where $N(r, t)$ denotes the probability of finding the diffusive entity at the position $r \in R^{d}$ (which may be discrete or continuous) at time $t$ if it was at the origin $r=0$ at time $\mathrm{t}=0$. Here $\mathrm{W}(\mathrm{r})$ is the fractional transition rate which measures the propensity for a displacemenr r. ${ }_{0} D_{t}^{\alpha}$ is the well-known Riemann-Liouville fractional derivative of order $\alpha$ defined in (Samko.Kilbas and Marichev,[11,p.37]; also see Kilbas, Srivastava and Trujillo,[12] )

$$
{ }_{0} D_{t}^{\alpha} N(x, t)=\frac{1}{\Gamma(n-\alpha)} \frac{d^{n}}{d t^{n}} \int_{0}^{t} \frac{f(x, \tau) d \tau}{(t-\tau)^{\alpha+1-n}}(n=[\alpha]+1), n \in N, \quad t>0,
$$

where $[\alpha]$ means the integral part of the number $\alpha$.

If we assume decoupling between $\mathrm{r}$ and $\mathrm{t}$ in the density function

,i.e. $N(r, t)=\xi(\mathrm{t}) \eta(\mathrm{r}),(1.1)$ then takes the form 


$$
{ }_{0} D_{t}^{\alpha} \xi(t) \eta(r)=\sum_{r^{\prime}} W\left(r-r^{\prime}\right) \xi(t) \eta(r),
$$

Since the time part of the above equation gives the fractal time evolution of fractional Brownian motion, we have

$$
{ }_{0} D_{t}^{\alpha} \xi(t)=-\lambda \xi(t)
$$

where $\lambda$ is the constant of separation, and the initial condition being

$$
\left.{ }_{0} D_{t}^{\alpha-1} \xi(t)\right|_{t=0}=\xi_{0} .
$$

In order to derive the solution of the equation (1.4), we apply the Laplace transform (Erdélyi et al, [13] ), defined by

$$
L\{N(t) ; s)=\int_{0}^{\infty} e^{-s t} N(t) d t=N^{\sim}(s),
$$

where $\operatorname{Re}(\mathrm{s})>0$, to its both sides, make use of following result, which gives the Laplace transform of the Riemann-Liouville fractional derivative [12], namely

$$
\begin{array}{r}
\left.\left\{{ }_{0} D_{t}^{\alpha} N(t) ; s\right\}=s^{\alpha} N^{\sim}(s)-\sum_{r=1}^{n} s^{r-1}\left[{ }_{0} D_{t}^{\alpha-r} N(t)\right]\right]_{t=0}, \\
(n-1<\alpha \leq n)(1.6)
\end{array}
$$

and apply the initial condition (1.5), we find that

$$
s^{\alpha} \xi^{\sim}(s)-\xi(0)=-\lambda \xi^{\sim}(s) .
$$

Solving for $\xi^{\sim}(s)$ and simplifying, we see that

$$
\xi^{\sim}(s)=\xi_{0} \sum_{r=0}^{\infty}(-1)^{r} \lambda^{r} s^{-\alpha-\alpha r},\left|\lambda / s^{\alpha}\right|<1 .
$$

The inverse Laplace transform of (1.8) yields the desired result

$$
\xi(t)=\xi_{0} t^{\alpha-1} E_{\alpha, \alpha}\left(-\lambda t^{\alpha}\right),
$$

where $E_{\alpha, \beta}(t)$ is the Mittag-Leffler function ( Erdélyi et al ,[6] ), defined by

$$
E_{\alpha, \beta}(z)=\sum_{n=0}^{\infty} \frac{z^{n}}{\Gamma(\alpha n+\beta)}(\alpha, \beta \in C ; \operatorname{Re}(\alpha)>0, \operatorname{Re}(\beta)>0) .
$$

By virtue of the relation givrn by Saxena, Mathai and Haubold [14,p.302,eq.(18)], (1.9) can be written in terms of the $\mathrm{H}$-function in the form

$$
\xi(t)=\xi_{0} t^{\alpha-1} H_{1,2}^{1,1}\left[\lambda t^{\alpha} \begin{array}{c}
(0,1) \\
(0,1) .(1-\alpha, \alpha)
\end{array}\right]
$$

$$
=\frac{\xi_{0} t^{\alpha-1}}{\alpha} H_{1,2}^{1,1}\left[\lambda^{1 / \alpha} t \mid \begin{array}{c}
(0,1 / \alpha) \\
(0,1 / \alpha) .(1-\alpha, 1)
\end{array}\right], \alpha>0(1,1
$$

where $H_{1,}^{1,1}[z]$ is the Fox's H-function, which is defined in terms of the Mellin-Barnes type integral in the form ( Mathai and Saxena,[7] ):

$$
\begin{aligned}
& H_{p, q}^{m, n}(z)=H_{p, q}^{m, n}\left[z \mid \begin{array}{c}
\left(a_{p}, A_{p}\right) \\
\left(b_{q}, B_{q}\right)
\end{array}\right] \\
& \left.=H_{p, q}^{m, n}|z| \begin{array}{l}
\left(a_{1}, A_{1}\right), \ldots,\left(a_{p}, A_{p}\right) \\
\left(b_{1}, B_{1}\right), \ldots,\left(b_{q}, B_{q}\right)
\end{array}\right]=\frac{1}{2 \pi i} \int_{\Omega} \Theta(\xi) z^{-\xi} d \xi,
\end{aligned}
$$

where

$$
\Theta(\xi)=\frac{\left[\prod_{i=1}^{m} \Gamma\left(b_{i}+B_{i} \xi\right)\right]\left[\prod_{j=1}^{n} \Gamma\left(1-a_{j}-A_{j} \xi\right)\right]}{\left[\prod_{i=m+1}^{q} \Gamma\left(1-b_{i}-B_{i} \xi\right)\right]\left[\prod_{j=n+1}^{p} \Gamma\left(a_{j}+A_{j} \xi\right)\right]}
$$

A detailed definition, properties, asymptotic expansion and a comprehensive account of the $\mathrm{H}$-function is available from the monographs written by Mathai and Saxena [7]. On using the asymptotic expansion of the $\mathrm{H}$-function for small and large values of the argument ( Mathai and Saxena,[7]), it is seen that

$$
\xi(t) \sim t^{\alpha-1} \text { as } t \rightarrow 0
$$

and

$$
\xi(t) \sim t^{-1} \text { as } t \rightarrow \infty .
$$

Remark 1. The results for the asymptotic estimate for the function $\xi(t)$ for small and large values of $\mathrm{t}$, as given by El-Wakil and Zahran [5,p. 1548 ] are erroneous. The correct values are given in the above equations (1.15) and (1.16) respectively. The method adopted here based on the Laplace transform for deriving the solution of (1.4) is faster in comparison to the Laplace- Mellin transform method employed in (El-Wakil and Zahran,[5] ) .

\section{Solution of Fractional Master Equation}

In this section, we will derive the solution of fractional master equation. The integral form of the fractional master equation is given by

$$
N(r, t)=\delta_{r 0}{ }^{+} 0 D_{t}^{-\alpha} \sum_{r^{\prime}} W\left(r-r^{\prime}\right) N(r, t),
$$

where $\delta_{r o}$ represents the initial condition at $\mathrm{t}=0$ and ${ }_{0} D_{t}^{-\alpha}$ is the Riemann-Liouville fractional integral, defined by 


$$
{ }_{0} D_{t}^{-\alpha} N(r, t)=\frac{1}{\Gamma(\alpha)} \int_{0}^{t}(t-u)^{\alpha-1} N(r, u) d u,(2.2)
$$

where $\operatorname{Re}(\alpha)>0$.

If we apply the Fourier transform, defined by

$$
N^{*}(k, t)=\int_{-\infty}^{\infty} \exp (i k r) N(r, t) d r,(k \geq 0),
$$

we find that

$$
N^{*}(k, t)=1+D_{t}^{-\alpha} W(k) N(k, t), \lim _{|r| \rightarrow \infty} N(r, t)=0 .
$$

Applying the Laplace transform to the above equation, it yields

$$
\begin{aligned}
& N^{*^{\sim}}(k, s)=\frac{s^{\alpha-1}}{\left(s^{\alpha}-W(k)\right)} \\
& =\sum_{r=0}^{\infty} s^{-\alpha r-1}(W(k))^{r},\left|\frac{W(k)}{s^{\alpha}}\right|<1 .
\end{aligned}
$$

Taking inverse Laplace transform of (2.5), it is found that

$$
\begin{aligned}
& N^{*}(k, t)=E_{\alpha, 1}\left(W(k) t^{\alpha}\right) \\
= & H_{1,2}^{1,1}\left[W(k) t^{\alpha} \mid \begin{array}{c}
(0,1) \\
(0,1),(o, \alpha)
\end{array}\right]
\end{aligned}
$$

The complete solution of (2.1) now follows by taking the inverse Fourier transform of the Mittag-Leffler function (2.6). Thus we finally obtain

$$
\mathrm{N}(\mathrm{r}, \mathrm{t})=\frac{1}{2 \pi} \int_{-\infty}^{\infty} \exp (-i k r) E_{\alpha, 1}\left(W(k) t^{\alpha}\right) d k
$$

For special values of $\mathrm{W}(\mathrm{k})$, one can obtain the inverse Fourier transform of (2.6) explicitly in a closed form . Setting $W(k)=-\eta|k|^{\gamma}, \gamma>0$, we see that in this case the required solution is obtained in a closed form in terms of the $\mathrm{H}$-function as

$$
N(r, t)=\frac{1}{\gamma|r|} H_{2,3}^{2,1}\left[\frac{|r|}{\eta^{1 / \gamma_{t} \alpha / \gamma}} \mid \begin{array}{c}
(1,1 / \gamma),(1, \alpha / \gamma),(1,1 / 2) \\
(1,1 / \gamma),(1,1),(1,1 / 2)
\end{array}\right],
$$

where we have employed the following result given by Haubold, Mathai and Saxena [17]:

$$
\begin{aligned}
& F^{-1}\left[E_{\alpha, \beta}\left(-\eta t^{\alpha} \mid k \gamma^{\gamma}\right) ; r\right] \\
& =\frac{1}{\gamma|r|} H_{2,3}^{2,1}\left[\frac{|r|}{\eta^{1 / \gamma} t^{\alpha / \gamma}} \mid \begin{array}{c}
(1.1 / \gamma),(\beta, \alpha / \gamma),(1,1 / 2) \\
(1,1 / \gamma),(1,1),(1,1 / 2)
\end{array}\right],
\end{aligned}
$$

(for $\beta=1$ ), where $\operatorname{Re}(\alpha)>0, \operatorname{Re}(\beta)>0, \gamma>0$.

When $\gamma=2$, then (2.9) gives the complete solution of the fractional master equation discussed by El-Wakil and Zahran
[5] in the form

$$
\begin{array}{r}
N(r, t)=\frac{1}{\gamma|r|} H_{2,3}^{2,1}\left[\frac{|r|}{\eta^{1 / 2} t^{\alpha / 2}} \mid \begin{array}{c}
(1,1 / 2),(1, \alpha / 2),(1,1 / 2) \\
(1,1 / 2),(1,1),(1,1 / 2)
\end{array}\right] \\
=\frac{1}{2|r|} H_{1,1}^{1,0}\left[\frac{|r|}{\eta^{1 / 2} t^{\alpha / 2}} \mid \begin{array}{c}
(1, \alpha / 2) \\
(1,1)
\end{array}\right]
\end{array}
$$

Remark 2. El-Wakil and Zahran [5] have obtained the solution for $W(k)=-\eta k^{2}$, in Fourier domain as

$$
N(k, t)=\frac{1}{\alpha} H_{1,2}^{1,1}\left[\left(\eta k^{2}\right)^{1 / \alpha} t \mid \begin{array}{c}
(0,1 / \alpha) \\
(0,1 / \alpha),(0,1)
\end{array}\right],
$$

which can be expressed in terms of the following series:

$$
N(k, t)=\sum_{r=0}^{\infty} \frac{(-1)^{r}}{\Gamma(\alpha r+1)}\left(\eta k^{2} t^{\alpha}\right)^{r},(\alpha \in C, \operatorname{Re}(\alpha)>0) \text {. }
$$

Remark 3. The result given by El-Wakil and Zahran [5,p.1548,eq..3.13) is incorrect. Its corrected version is given by (2.14). As $t \rightarrow \infty$, then considering the asymptotic expansion of the $\mathrm{H}$ - function for large $\mathrm{t}$, we find that

$$
N(k, t) \sim t^{-\alpha} \text {,as } t \rightarrow \infty \text {. }
$$

\section{A Generalized Fractional Diffusion Equation}

In this section, we give an alternative shorter method for deriving the solution of a generalized diffusion equation investigated by Hilfer [16] (2000). Below we define the (right/left-sided) fractional derivative of order $0<\alpha<1$ and type $0 \leq \beta \leq 1$ with respect to $\mathrm{x}$, introduced by Hilfer [16] in the following form:

$$
D_{a+}^{\alpha, \beta} f(x)=\left( \pm I_{a \pm}^{\beta(1-\alpha)} \frac{d}{d x}\left(I_{a \pm}^{(1-\beta)(1-\alpha)} f\right)\right)(x),(3.1
$$

which holds for functions for which the right hand side of (3.1) exists. The Riemann-Liouville fractional derivative $D_{a \pm}^{\alpha}:=D_{a \pm}^{\alpha}$ corresponds to $a<-\infty$ and type $\beta$. The Laplace transform of the above operator is given by

$$
\begin{aligned}
& L\left\{D_{a \pm .}^{\alpha, \beta} f(x) ; s\right\} \\
& =s^{\alpha} L\{f(x), s\}-s^{\beta(\alpha-1)}\left(D_{a+}^{(1-\beta)(1-\alpha)} f\right)(0+),
\end{aligned}
$$

where the initial value $\left(D_{a+}^{(1-\beta)(\alpha-1), 0}\right)(0+)$ is the

Riemann-Liouville fractional derivative as $t \rightarrow 0$.

Theorem 2. Consider the Cauchy problem for the 
differential equation of fractional order

$$
D_{0+}^{\alpha, \beta} G(\vec{r}, t)=\eta \Delta G(\vec{r}, t),
$$

where $G ; R^{d} \times R^{d} \rightarrow R, \Delta$ is the Laplacian with initial condition

$$
I_{0+}^{(1-\beta)(1-\alpha)} G(\vec{r}, 0+)=G_{0 r} \rightarrow=g_{0} \delta(\vec{r}),
$$

Here $\eta$ is a diffusion constant and $\delta(r)$ is the Dirac measure at the origin. Then (3.3) has the solution given by

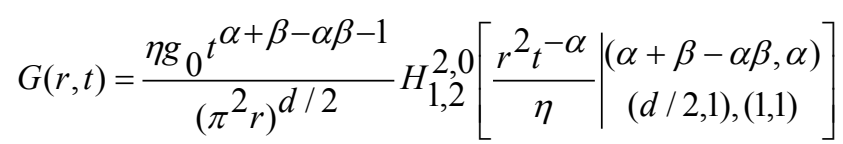

Proof. By the application of the Laplace transform and the Fourier transform, defined by

$$
F\{g(r) ; k\}=\int_{R} d \exp (i k \rightarrow . r \rightarrow) g(r \rightarrow(d r \rightarrow),
$$

We find that

$$
G(k \rightarrow, s)=\frac{g_{0}{ }^{\beta(\alpha-1)}}{\eta k^{\rightarrow 2}+{ }^{\alpha}},
$$

By virtue of the formula given by Saxena ,Mathai and Haubold,[8,p. 293,eq.(41)], the inverse Laplace transform of (3.7) gives

$$
G(k \rightarrow, t)=g_{0} t^{(1-\beta)(\alpha-1)} E_{\alpha \alpha+\beta(1-\alpha)}\left(-\eta k^{\rightarrow 2} t^{\alpha}\right),
$$

In order to invert (3.7), it is convenient to first invert the Fourier transform and then Laplace transform. By making use of the formula given in (Samko,Kibas and Marichev, [11, p.538, eq.(27.1)]; also see Saxena,Mathai and Haubold [16])

$$
\begin{aligned}
& (2 \pi)^{-d / 2} \int e^{i k \rightarrow} . r \rightarrow\left(\frac{|r \rightarrow|}{\mu}\right)^{1-d / 2} K\left(\frac{d-2}{2}\right)(\mu|r \rightarrow|) d r \rightarrow \\
& =\frac{1}{(k \rightarrow)^{2}+\mu^{2}},
\end{aligned}
$$

It readily follows that

$$
\begin{aligned}
& G(r \rightarrow, t)= \\
& g_{0}(2 \pi \eta)^{-d / 2}\left(\frac{r}{\eta^{1 / 2}}\right)^{1-\frac{d}{2}}{ }_{s}^{\beta(\alpha-1)+\alpha(d-2) / 4} K\left(\frac{d-2}{2}\right)\left(\frac{r s^{\alpha / 2}}{\eta^{1 / 2}}\right),
\end{aligned}
$$

where $r=|r \rightarrow|$ and $K_{V}($.$) is the modified Bessel$ function of the second kind or MacDonald function. If we take the inverse Laplace transform of (3.9) with the help of the following formula given by Saxena, Mathai and Haubold [8,p. 291,eq.(20)]

$$
\begin{aligned}
& 2 L^{-1}\left\{s^{-\rho} K_{V}\left(z s^{\sigma}\right) ; t\right\} \\
& =t^{\rho-1} H_{1,2}^{2,0}\left[\frac{z^{2} t^{-2 \sigma}}{4} \mid \begin{array}{c}
(\rho .2 \sigma) \\
(v / 2,1),(-v / 2,1)
\end{array}\right],
\end{aligned}
$$

where $\operatorname{Re}(\mathrm{s})>0, \operatorname{Re}\left(z^{2}\right)>0$. we obtain

$$
\begin{aligned}
G(r \rightarrow, t) & =g_{0}(2 \pi \eta)^{-d / 2}\left(\frac{r}{\eta^{1 / 2}}\right)^{1-\frac{d}{2}(1-\alpha) \beta-\alpha\left(\frac{d-2}{4}\right)-1} \\
& \times H_{1,2}^{2,0}\left[\frac{r^{2} t^{-\alpha}}{4 \eta} \mid \begin{array}{c}
\alpha / 2+\beta-\alpha \beta-\alpha d / 4, \alpha) \\
\left(\frac{d-2}{4}, 1\right),\left(\frac{2-d}{4}, 1\right)
\end{array}\right] .(3.12)
\end{aligned}
$$

If we insert the expression $\left(\frac{r^{2} t^{-\alpha}}{4 \eta}\right)^{\frac{d+2}{4}}$ inside the $\mathrm{H}$-function in the above equation by means of the formula ( Mathai and Saxena,[7 ,p. 4 , eq, (1,2.4 )]), namely

$$
z^{\kappa} H_{p, q}^{m, n}\left[z \mid \begin{array}{c}
\left(a_{p}, A_{p}\right) \\
\left(b_{q}, B_{q}\right)
\end{array}\right]=H_{p, q}^{m, n}\left[z\left[\begin{array}{c}
\left(a_{p}+\kappa A_{p}, A_{p}\right) \\
\left(b_{q}+\kappa B_{q}, B_{q}\right)
\end{array}\right] .\right.
$$

we arrive at the desired result (3.5).

Remark 4. It is interesting to observe that for $\beta=1, \quad(3.12)$ reduces to a result given by Kochbei [18], in which case $G(r \rightarrow, t)$ is a probability density function.

\section{Discussion}

In this paper, we have used a composite fractionl derivative which includes Riemann -Liouville and Caputo fractional derivative operators. Further due to presence of the generalized Mittag -Leffler function and Fox's H-function in the solution, it can be computed numerically, thus enhancing the utility of the derived results .

\section{Acknowledgements}

The author is grateful to the referee for giving some useful suggestions which led to the present form of the paper.

\section{REFERENCES}

[1] R.Hilfer. and L. Anton. Fractal master equations and fractal time random walks, Phys. Rev. Vol.E 51, No.2. R848- R851, 1995. 
[2] G.Jumarie.Fractional Master equation: non -standard analysis and Liouville - Riemann derivative, Chaos, Solitons and F, Vol.12, <No.13, 2577-2587, 2001.

[3] G.Pagnini,A.Mua and F. Mainardi,, Generalized fractional Master equation for self -similar stochastic processes Modelling Anomalous diffusion, InternationalJournal of Stochastic Analysis, Vol. 2012, Article ID 427383 (14 pages) Doi:10 . 1155/2012/427383,2012.

[4] R. Metzler, E.Barkai and J. Klafter, Deriving fractional Fokker - Planck equations from a generalized Master equation,, Europhys. Lett. 46 431(Volume 46, No. 4), (1999), Doi: 101209 /ep[f] 1999- 00279-7.

[5] S.A. El- Wakil and M.A.Zahran Fractional integral representation of master equation, Chaos,Solitons and Fractals, Vol.10, 1545-1548, 1999.

[6] A.Erdélyi.,W. Magnus., F. Oberhettinger and F.G.Tricomi. Higher Transcendental Functions, Vol. 3, McGraw- Hill, New York, Toronto and London, 1955..

[7] A.M.Mathai and R.K.Saxena. The H-function with Applications in Statisticsand Other Disciplines,John Wiley and Sons, Inc. New York, 1978.

[8] R.K.Saxena., A.M.Mathai., and H.J.Haubold. Fractional reaction-diffusion equations, Astrophysics and Space Science 305,289-296, 2006.

[9] R. Hilfer. In Random Magnetism and High Temperature Super-conductivity, edited by W. Beyermann ,World Scientific,Singapore, 1994.
[10] R. Hilfer. Chaos, Solitons \& Fractals Vol.5, p.1475, 1995.

[11] S.G.Samko., A.A.Kilbas and O.I.Marichev, O. Fractional Integrals and Derivatives, Theory andApplivations, Gordon and BreachPublishers, New York, 1993.

[12] A,A,Kilbas., H.M.Srivastava and J.J.Trujillo. Theory and Applications of Fractional Differential Equations, Elsevier, Amsterdam. 2006

[13] A.Erdélyi.,W.Magnus.,F. Oberhettinger,F. and F.G. Tricomi, Tables of Integral Transforms, Vol. 1, McGraw-Hill, New York. , Toronto and London. 1954.

[14] R.K.Saxena.,A.M.Mathai and H.J.Haubold. Unified fractional kinetic equations and a fractional diffusion equation, Astrophysics and Space Science, 290, 299-310, 2004.

[15] R.K.Saxena.,A.M.Mathai.,and H.J.Haubold. Solution of certain fractional kinetic equations and a fractional diffusion equation, International Journal of Scientific Research, Vol. 17,1-18,2008.

[16] R. Hilfer. (ed..) .Fractional Time Evolution in : Applications of Fractional Calculus in Physics, World Scientific, Sinapore, 2000.

[17] H.J.Haubold.,A.M.Mathai and R.K.Saxena, Solutions of fractional reaction -diffusion equations in terms of the H-function, Bull.Astr. Soc., india, Vol. 35, 681-689, 2007.

[18] A.N.Kochubei. Diffusion of fractional order, Differential Equations Vol.26, 485-492. 1990. 